\title{
Drug-Drug Interactions among Hemodialysis Patients
}

\author{
Shireen I Hijazeen*, Tamara S Altawisi, Alaa M Aqarbeh, Isra'a H Alawneh, Lana A AlIssa
}

The Royal Medical Services Amman/ Jordan

DOI: $10.36347 /$ sajp.2020.v09i01.007

| Received: 02.12.2019 | Accepted: 09.12.2019 | Published: 22.01.2020

*Corresponding author: Shireen I Hijazeen

Abstract

Original Research Article

Chronic kidney disease (CKD) increased and became a public health problem. Patients who have end stage renal disease (ESRD) and who receive hemodialysis (HD) are expected to be given 10 to 12 medications daily and many of these medications need multiple doses per day. Owing to poly-pharmacy, recurrent medication modifications on HD versus non- dialysis days, therapeutically unstable nature of the illness and limited life styles, these patients is at high risk for having drug related problems (DRPs).This study was conducted to evaluate potential DDIs among HD patients.An observational- retrospective study was carried out; it included ESRD patients undergoing maintenance hemodialysis in the HD unit in the King Hussein Medical Centre (KHMC) at the Royal Medical Services (RMS) in Jordan/Amman. The study included 300 patients, a total of 930 potential interactions were identified in 277 (92.3\%) patients. Patients were prescribed $8-12$ drugs with a mean $( \pm$ SD) of $8.26 \pm 3.56$.

Keywords: Drug, Hemodialysis, Interaction, Chronic Kidney Disease.

Copyright @ 2020: This is an open-access article distributed under the terms of the Creative Commons Attribution license which permits unrestricted use, distribution, and reproduction in any medium for non-commercial use (NonCommercial, or CC-BY-NC) provided the original author and source are credited.

\section{INTRODUCTION}

Chronic kidney disease (CKD) represents a major public health problem in developed and developing countries. It is estimated that about $10 \%$ of the adult U.S. population is suffering from CKD. CKD defined as the presence of kidney damage or decreased kidney function for three or more months [1].

In Jordan; CKD has been growing rapidly in recent years and more than 746 people per million were receiving hemodialysis in 2012, an increase from the previous year of $1.1 \%$. CKD and end-stage renal disease (ESRD) are associated with an increased risk of mortality and morbidity, and reduced average life expectancy [2].

As outlined in table (1), kidney failure is defined as either (1) GFR below $15 \mathrm{ml} / \mathrm{min}$ accompanied with signs or symptoms of uremia in most of the time or (2) the need to initiate renal replacement therapy to treat complications associated with severe reduction in GFR. Some patients start HD before the GFR declines below $15 \mathrm{ml} / \mathrm{min}$ due to symptoms of uremia [3, 4]. End stage kidney disease is an administrative term that is used in the United States (US) based on the conditions for payment for health care by the Medicare ESRD Program. It includes patients on HD or those who have undergone transplantation irrespective of the level of GFR. Kidney Disease Outcomes Quality Initiative Work Group definition, as stated in 2002 clinical practice guidelines, differs from ESRD definition in two ways [5].

According to K/DOQI Work Group, not all patients with GFR below $15 \mathrm{ml} / \mathrm{min}$ are treated with dialysis or transplantation, but they are considered to have renal failure. Moreover, patients who have undergone transplantation have higher GFR and better average health outcomes and thus aren't included in the definition of kidney failure unless they have GFR below $15 \mathrm{ml} / \mathrm{min}$ or resumed dialysis [4].

Table-1: National Kidney Foundation/ Kidney Disease Outcomes Quality Initiative classification of CKD:

\begin{tabular}{|l|l|l|}
\hline Stage & Description & GFR(ml/min/1.73m \\
\hline $\mathbf{1}$ & Kidney damage with normal or $\uparrow$ GFR & $\geq 90$ \\
\hline $\mathbf{2}$ & Kidney damage with normal or $\downarrow$ GFR & $60-89$ \\
\hline $\mathbf{3}$ & Moderate $\downarrow$ GFR & $30-59$ \\
\hline $\mathbf{4}$ & Severe $\downarrow$ GFR & $15-29$ \\
\hline $\mathbf{5}$ & Kidney failure & $<15$ (or dialysis) \\
\hline
\end{tabular}


Patients who have ESRD and who are on regular HD are administered 10 to 12 drugs per day and numerous of these drugs necessitate frequent doses [5]. Owing to multiple drugs prescribing patients on maintenance HD are at high risk for having drug related problems (DRPs) [6].

The health care teams must remember that pharmacological choices must be well- adjusted with the possible risk of numerous medication uses. The possible hazards include side effects, unsuitable dosing schedules, medication-disease interactions and drugdrug interactions (DDIs) [7]. Although DDIs are measured as preventable DRPs, the literature showed that up to $12 \%$ of patients had symptoms related to DDIs and constitute for around $4.1 \%$ of hospital admissions [8].

It is vital to keep in mind that knowing possible DDIs in a patient does not mean he will undergo a clinically adverse effect, occasionally additional observing is the solitary action required. Observing of possible DDIs may advance the value of recommending and dispensing drugs and it might be the foundation for instruction attentive on proper prescribing [9].

It is expected that this study will aid increase alertness of the significance of drug observing and review in HD patients.

The aims of this study is to study was designed to assess the prescribed medications and pattern of distribution for potential DDIs among HD patients attending the hemodialysis unit at in the King Hussein Medical Centre (KHMC) at the Royal Medical Services (RMS) in Jordan/Amman.

\section{METHODS}

\section{Study Design}

For this study, a non-experimental, descriptive, cross-sectional design will be used to meet purpose of the study. The researcher chosen this design because it attain the aim of the study and is time-efficient. An observational- retrospective study was carried out; it included ESRD patients having maintenance HD in the King Hussein Medical Centre (KHMC) at the Royal Medical Services (RMS) in Jordan/Amman, between July and September 2018. The data collection form was accomplished by retrieving from the patients' medical records.

The study protocol was permitted by obtaining the ethical approval from the IRB committee at the RMS before the start of this study. All participants' rights were ensured based on ethical principles of respect for human dignity, privacy, confidentiality and autonomy. Participation in the study will be voluntarily and anonymously. In addition, each patient was informed regarding his/her right of withdrawal at any time. All participants were informed regarding the purpose of the study. The study procedure was explained for each participant, and informed verbal consent from each eligible patient obtained before start the data collection. After data collection, all data was coded and entered to a password protected computer and accessed only by the researcher.

\section{Sample Size}

The population is the all possible participant from which the researcher is going to pick up the sample or the entire aggregate of cases in which the researcher is interested.

A total of $300 \mathrm{HD}$ patients were included in this study.

\section{DATA COLLECTION}

Patient's medical profiles were revised. All of the information was organized for analysis. A software program for drug interactions by Medscape was used for screening the possible DDIs.

\section{STATISTICAL ANALYSIS}

Statistical analysis was performed using Statistical Package for Social Sciences (SPSS version 22). Mean \pm standard deviation was computed for continuous data. Frequencies and percentages were calculated for categorical variables.

\section{RESULTS}

Total number of patients who were receiving HD in KHMC was 350 patients, of whom 312 patients were eligible, 12 patients refused to participate in the study due to inconvenience. A total number of 300 patients of both genders were enrolled into the study.

\section{Patients Demographics}

The study population ranged in age from 20 to 79 years with a mean \pm SD $53.6 \pm 16.8$. Among the screened patients, $191(63.7 \%)$ were males. The majority of the patients, 200 patients $(66.7 \%)$, had normal body weight, while 51 patients $(17 \%)$ were overweight. The main causes of ESRD in this population were diabetes mellitus $(37.5 \%)$ and HTN $(32.1 \%)$. Larger part of the patients completed bachelors $(28.6 \%)$ and only small number of patients were illiterate $(8.9 \%)$. Education level was obtained directly from the patient and based on the last grade reached by the patient. Patients received maintenance HD for a mean of 6.7 years.

Patients varied in the number of HD sessions, as 166 patients $(55.3 \%)$ received 3 sessions per week and 134 patients $(44.7 \%)$ received 2 sessions per week. Hours of dialysis per week for all patients ranged from 7 to 11 hours with a mean \pm SD $11.1 \pm 1.3$ hours. All patients were prescribed blood flow rate of $289 \pm 38.1$ $\mathrm{ml} / \mathrm{min}$. 


\section{Medications in HD Patients}

Throughout the study period, a total of 35 different drugs were used by the patients. The patients were taking a minimum of 4 and a maximum of 12 medications, with a mean $( \pm \mathrm{SD})$ of $5.16 \pm 3.21$. The most commonly prescribed medications were calcium carbonate being used by 238 (79.3\%). Followed by Alfacalcidol, Ferrous sulphate, Acetyl salicylic acid and Nifedipine respectively.

Table-2: The most common medications used by the study population

\begin{tabular}{|l|c|}
\hline Medication & Number of patients \\
\hline CaCO3 & $238(79.3 \%)$ \\
\hline Alfacalcidiol & $201(67 \%)$ \\
\hline Acetyl salicylic acid & $189(63 \%)$ \\
\hline Ferrous sulphate & $176(58.6 \%)$ \\
\hline Nifedipine & $175(58.3 \%)$ \\
\hline Amlodipine & $164(54.6 \%)$ \\
\hline Frusemide & $160(53.6 \%)$ \\
\hline Enalapril & $158(52.3 \%)$ \\
\hline Candesartan & $151(50.3 \%)$ \\
\hline Bisoprolol & $149(49.6 \%)$ \\
\hline Atenolol & $139(46.3 \%)$ \\
\hline Carvedilol & $135(45 \%)$ \\
\hline Hydralazine & $122(40.6 \%)$ \\
\hline Doxazocin & $115(38.3 \%)$ \\
\hline Tinzaparin & $80(26.6 \%)$ \\
\hline Subcutaneous insulin & $77(25.6 \%)$ \\
\hline Famotidine & $75(25 \%)$ \\
\hline Omeprazole & $66(22 \%)$ \\
\hline Simvastatin & $51(17 \%)$ \\
\hline Glimepiride & $45(15 \%)$ \\
\hline Carbamazepine & $33(11 \%)$ \\
\hline Nitrates & $31(10.3 \%)$ \\
\hline Others 1 & $26(8.6 \%)$ \\
\hline
\end{tabular}

Allopurinol, Alendronate Na, Gemfibrizoil, Multi vitamins, Tamsolucin, Warfarin

\section{Potential Drug-Drug Interactions (DDIs)}

Amongst 300 patients, $289(96.3 \%)$ patients had at least one possible DDI. Among the 289 patients who had possible interactions, a total of 932 interactions were recognized. According to risk rating classification, $566(60 \%)$ were serious, $134(14.4 \%)$ were significant, and $230(24.7 \%)$ were minor. The most prevalent interaction in $114(41.5 \%)$ patients was calcium carbonate with Nifedipine (Calcium salts may diminish the therapeutic effect of Calcium Channel Blockers) followed by aspirin with calcium carbonate in $76(27.6 \%)$ cases (Antacids may decrease the serum concentration of Salicylates). A total of 994 DDIs were found in the prescriptions of 300 patient's medical profiles that were evaluated. Majority of the DDIs (64.14\%) were significant. Table 4 shows the top ten possible DDIs.

Table-3: Drug-Drug Interactions Based on Medscape Classifications

\begin{tabular}{|c|c|}
\hline Category & $\mathbf{N}(\boldsymbol{\%})$ \\
\hline Serious & $566(60 \%)$ \\
\hline Significant & $134(14.4 \%)$ \\
\hline Minor & $230(24.7 \%)$ \\
\hline
\end{tabular}

Table-4: Top Ten DDIs

\begin{tabular}{|c|c|c|}
\hline No & Drug-Drug interaction & Risk rating \\
\hline 1 & Gemfibrozil + Simvastatin & Significant \\
\hline 2 & Aspirin + Enalapril & Serious \\
\hline 3 & Amlodipine + Simvastatin & Serious \\
\hline 4 & Aspirin + Glimepiride & Serious \\
\hline 5 & Atenolol + Telmisartan & Serious \\
\hline 6 & Calcium Carbonate + Atenolol & Serious \\
\hline 7 & Calcium Carbonate + Nifedipine & Serious \\
\hline 8 & Gemfibrozil + Glimepiride & Serious \\
\hline 9 & Glimepiride + Insulin & Serious \\
\hline 10 & Calcium Carbonate + Aspirin & Minor \\
\hline
\end{tabular}

\section{DISCUSION}

Patients with stage 1 to $5 \mathrm{CKD}$ and those undergoing dialysis are at extremely high risk for drug therapy problems (DTPs) since they are prescribed an average of 12 medications and present around six comorbidities [10]. Professional health care providers of physicians, dieticians, clinical pharmacists and nurses have the target of preventing disease progression and correcting co-morbid conditions in CKD and ESRD patients. As specialists in pharmacotherapy, clinical pharmacists have the role in providing patient care and communicate with other members of the health care team, managing various, often unmet requirements for pharmacotherapy optimization. Ideally, this can be achieved through a preventive, rather than a reactive process. Various Evidences from the literature support the role of clinical pharmacists in many disease areas and confirms the positive patient impacts and improvement of care that result[11].

In this research, the $300 \mathrm{HD}$ patients were prescribed 35 different drugs with a mean $( \pm$ SD) of $5.16 \pm 3.21$. The number of drugs in this research is near to a Japanese research where the mean was about 6.1 drugs. The most prevalent prescribed medications in current study were calcium carbonate, followed by alfacalcidol, ferrous sulphate, acetylsalicylic acid and Nifedipine. An observational research was performed in HD unit in a private hospital in India, the five most commonly prescribed medications were Aspirin, Insulin, Amlodipine, calcium carbonate, and alfacalcidol [12]. Owing to this poly- pharmacy, HD patients are at higher risk for having DRPs including DDIs[13].

In the current study, the prevalence of possible DDIs was $96.3 \%$, this is a high percent. This is consistent with a research from Norway where the possible DDIs among HD patients touched 84.9\%[14].

There are various ways that can be applied to categorize DDIs [13]. In the present study, Medscape ${ }^{\circledR}$ was applied for this aim. Most of the possible DDIs were at serious- risk rating which means that monitoring is required. In a cross sectional study that was conducted in a three American hospitals [14], each combination of given medication was examined by means of the Micromedex ${ }^{\circledR}$ system to search for 
possible DDIs, among the 673 patients, 503 DDIs were recognized[15].

Health care providers including pharmacists must be more conscious of these possible DDIs and they should cooperate to improve instructive databases and expand patients' counseling to elude inappropriate usage of drugs[16].

\section{LIMITATIONS}

- There was no routine or weekly clinical round performed by the nephrologist in the dialysis unit, which made it difficult for the clinical pharmacist to give recommendations as efficient as hoped.

- Poor documentation and missing data in the medical profiles hindered adequate collection of information.

\section{CONCLUSION}

The prevalence of possible DDIs among HD patients is very common; they are very predictable and rely on the number of drugs given to the patients. Several of these DDIs are considered as avoidable, so detection for possible DDIs and checking regularly must occur regularly before recommending any drug to improve quality of prescribing and dispending. To manage TRPs, each HD unit should have a clinical pharmacist as a member of the health care team to provide medical care to HD patients. Services administered by a clinical pharmacist have been shown to be cost-effective and associated with improvement of the quality of life.

\section{REFERENCES}

1. Levey AS, Eckardt KU, Tsukamoto Y, Levin A, Coresh J, Rossert J, De Zeeuw D, Hostetter TH, Lameire N, Eknoyan G. Definition and classification of chronic kidney disease: a position statement from Kidney Disease: Improving Global Outcomes (KDIGO) Kidney

Int. 2005;67(6):2089-100.

2. Wen CP, Cheng TY, Tsai MK, Chang YC, Chan HT, Tsai SP, Chiang PH, Hsu CC, Sung PK, Hsu YH. All-cause mortality attributable to chronic kidney disease: a prospective cohort study based on 462293 adults in Taiwan. Lancet. 2008;371(9631):2173-82.

3. Huang CC, Chan WL, Chen YC, Chen TJ, Chung CM, Huang PH, Lin SJ, Chen JW, Leu HB. The beneficial effects of statins in patients undergoing hemodialysis. Int J Cardiol. 2013;168(4):4155-9.

4. Barsoum RS. Chronic kidney disease in the developing world. N Engl J Med. 2006;354(10):997-9.

5. Khader MI, Snouber S, Alkhatib A, Nazzal Z,
Dudin A. Prevalence of patients with end-stage renal disease on dialysis in the West Bank, Palestine. Saudi J Kidney Dis Transpl. 2013;24(4):832-7.

6. Rama M, Viswanathan G, Acharya LD, Attur RP, Reddy PN, Raghavan SV. Assessment of drug-drug interactions among renal failure patients of nephrology ward in a South Indian Tertiary Care Hospital. Indian J Pharm Sci. 2012;74(1):63-8.

7. LaFleur J, McBeth C, Gunning K, Oderda L, Steinvoort C, Oderda GM. Prevalence of drugrelated problems and cost-savings opportunities in medicaid high utilizers identified by a pharmacistrun drug regimen review center. J Manag Care Pharm. 2006;12(8):677-85.

8. Mahmood M, Malone DC, Skrepnek GH, Abarca J, Armstrong EP, Murphy JE, Grizzle AJ, Ko Y, Woosley RL. Potential drug-drug interactions within Veterans Affairs medical centers. Am J Health Syst Pharm. 2007;64(14):1500-5.

9. Merlo J, Liedholm H, Lindblad U, Bjorck-Linne A, Falt J, Lindberg G, Melander A. Prescriptions with potential drug interactions dispensed at Swedish pharmacies in January 1999: cross sectional study. BMJ. 2001;323(7310):427-8.

10. Eiam-Ong S, Sitprija V. Comorbidities in patients with end-stage renal disease in developing countries. Artificial organs. 2002;26(9):753-6.

11. Schwedt E, Fernandez J, Gonzalez F, Mazzuchi N. Renal replacement therapy in Latin America during 1991-1995. Latin American Registry Committee. Transplantation proceedings. 1999;31(7):3083-4.

12. Tozawa $M$, Iseki $K$, Iseki $C$, Oshiro $S$, Higashiuesato Y, Yamazato M, Tomiyama N, Tana $\mathrm{T}$, Takishita S. Analysis of drug prescription in chronic haemodialysis patients. Nephrol Dial Transplant. 2002;17(10):1819-24.

13. Al-Ramahi R. Medication prescribing patterns among chronic kidney disease patients in a hospital in Malaysia. Saudi J Kidney Dis Transpl. 2012;23(2):403-8.

14. Manley HJ, Cannella CA, Bailie GR, St Peter WL. Medication-related problems in ambulatory hemodialysis patients: a pooled analysis. Am J Kidney Dis. 2005;46(4):669-80.

15. Bajait CS, Pimpalkhute SA, Sontakke SD, Jaiswal KM, Dawri AV. Prescribing pattern of medicines in chronic kidney disease with emphasis on phosphate binders. Indian J Pharmacol. 2014;46(1):35-9.

16. Grabe DW, Low CL, Bailie GR, Eisele G. Evaluation of drug-related problems in an outpatient hemodialysis unit and the impact of a clinical pharmacist. Clin Nephrol. 1997;47(2):11721. 